\title{
EVOLUCIÓN DE LOS SERVICIOS DE ORIENTACIÓN EN LA COMUNIDAD AUTÓNOMA DE GALICIA SITUACIÓN ACTUAL
}

\author{
DEVELOPMENT OF COUNSELLING SERVICES IN THE \\ AUTONOMOUS COMMUNITY OF GALICIA
}

\author{
Elena Menéndez Martín* \\ Consellería de Educación y Ordenación Universitaria de la Xunta de Galicia
}

\section{RESUMEN}

El artículo contiene el resultado de un estudio centrado en la evolución de los servicios de orientación en la Comunidad Autónoma de Galicia.

Se han estudiado cada uno de los servicios en todos los aspectos, tanto de funciones, convocatorias, selección y otros, pretendiendo dar información por su valor en sí o como futuras comparaciones con las demás Comunidades Autónomas.

Palabras clave: S.O.E.V., E.Ps.A., E.M. funciones, convocatorias y selección, horaios y vacaciones.

\section{ABSTRACT}

This article is about the results of studying the evolution of the guidance services in Galicia region. All the services have been studied in all their aspects: duties, summons,selection and anothers one, intending to give information by its own vlues or comparing it in the future with others regions.

Key words: S.O.E.V., E.Ps.A., E.M., dutier, summons and selection, timetable and holidays.

\section{Introducción}

Estamos viviendo un momento de gran importancia en la enseñanza, en general y en el campo de la orientación educativa, en particular.

* Doctora en Pedagogía. Inspectora de Educación de la Consellería de Educación y Ordenación Universitaria de la Xunta de Galicia, en La Coruña. Trayectoria profesional en orientación: Miembro de los E.Ps.A; Coordinadora de E.Ps.A; Inspectora-Directora de los E.Ps.A; Inspectora-Coordinadora de los E.O.E. Línea de investigación: Orientación y servicios en las distintas Comunidades Autónomas. Lugar de trabajo: Inspección de Educación. La Coruña. Domicilio: Alfredo Vicenti 8-3º 15004 La Coruña. 
La orientación se enmarca en la Reforma y, en particular, en el contexto del nuevo currículum establecido para los diferentes niveles, acompañándola como elemento de apoyo al currículum, a la calidad de la enseñanza y a la personalización de los aprendizajes.

La necesidad de la orientación, que no es nueva ya que cuenta con muchos años de historia en algunos países, hay que añadirle los cambios experimentados en los últimos años, que no puede referirse a una intervención puntual, si no que es necesario concebirla como una acción continuada a lo largo del proceso educativo.

\section{Evolución de los servicios de orientación desde el traspaso de competencias}

Desde la Ley Orgánica del 81, por la que se produce el traspaso de competencias, no ha habido excesiva legislación en esta Autonomía. La adecuación de los S.O.E.V. y los E.M. a los E.Ps.A. se describe a continuación

\section{Servicios que se traspasan}

Por el Real Decreto 1763/1982, de 24 de julio, se hace el traspaso de competencias plenas en materia de educación a la Xunta de Galicia. Una Resolución de la Dirección General de Educación Básica de la Consellería de Educación del 15 de diciembre de 1982 (D.O.G. de 14 de febrero de 1982) fija la organización y funcionamiento de los S.O.E.Vs. en la Comunidad Autónoma, en el ámbito de la E.G.B.

Por otra Resolución de 11 de mayo de 1983 (D.O.G. de 17 de junio) de la Dirección General de Educación Básica, se regula la composición, organización y funciones de los Equipos Multiprofesionales en el ámbito de la E.G.B.

\section{Servicios de Orientación Escolar y Vocacional. (S.O.E.V.)}

Normativa

\begin{tabular}{|l|l|}
\hline \multicolumn{2}{|c|}{ SOBRE } \\
\hline \multicolumn{1}{|c|}{ Organización, funciones y regulación } & \multicolumn{1}{c|}{ Convocatoria de plazas } \\
\hline $\begin{array}{l}\text { - Resolución de 15/12/82 (D.O.G. de 14/2/83) } \\
\text { - Orden de 26/3/85 (D.O.G. de 11/4/85) }\end{array}$ & $\begin{array}{l}\text { - Resolución de } 10 / 9 / 82 \text { (D.O.G. de 2/10/82) } \\
\text { - Resolución de 8/2/83 (D.OG. de 19/2/83) }\end{array}$ \\
& - Resolución de 16/1/84 (D.O.G. de 31/1/84) \\
\hline
\end{tabular}

\section{Funciones}

- No difieren sustancialmente de las establecidos con anterioridad, con carácter estatal.

- Orientación escolar, personal y vocacional.

- Asesorar al profesorado. 
- Información profesional.

- Localización de alumnos de Educación Especial.

\section{Convocatoria y selección}

Requisitos para el acceso

- Pertenecer al cuerpo de Profesores de Educación General Básica y poseer algunas de las titulaciones siguientes:

a) Licenciado en Filosofía y Letras (Sección de Pedagogía) o en Filosofía y Ciencias de la Educación (Sección de Pedagogía).

b) Licenciado en Filosofía y Letras (Sección Psicología) o en Filosofía y Ciencias de la Educación (Sección Psicología).

c) Licenciado en cualquier Facultad o Escuela Técnica Superior y Diplomado en Psicología Escolar.

\section{Equipos Multiprofesionales}

\section{Normativa}

- Orden de 9 de septiembre de 1982 (B.O.E. de 15 de septiembre).

- Resolución de 11 de mayo de 1983 (D.O.G. de 17 de junio.

\section{Funciones}

- La detección precoz de las deficiencias, anomalías y trastornos.

- La valoración pluridimensional de las necesidades y capacidades del sujeto de Educación Especial.

- La elaboración de programas de desarrollo individual en los que se fijen los objetivos, contenidos, metodologías y recursos necesarios para la solución de la problemática específica de cada niño.

- El seguimiento del programa de desarrollo individual en colaboración con el profesor de los centros educativos.

- La acción preventiva en el medio social, familiar y escolar.

- Contribuir a la elaboración del diseño del cuadro de necesidades concretas en el sector de la Educación Especial, elevando, a través del Inspector Ponente, a la Dirección General los presupuestos y posibles soluciones técnicas o sugerencias prácticas.

\section{Composición}

Estarán constituidos por:

- Dos Licenciados en Pedagogía. 
- Dos Licenciados en Psicología.

- Un Licenciado en Medicina.

- Dos Asistentes Sociales.

- Un Auxiliar Administrativo.

\section{Convocatoria y selección}

La selección se hará mediante pruebas de acceso y sus condiciones administrativas se reglamentarán mediante contratos laborales.

\section{Creación de los Equipos Psicopedagógicos de Apoyo (E.Ps.A.)}

Se decide, finalmente, unificar los S.O.E.V. y los E.M., creándose los Equipos Psicopedagógicos de Apoyo para el asesoramiento a los centros educativos en el ámbito del diagnóstico, orientación e integración de alumnos.

La experiencia acumulada a lo largo de los dos últimos años de funcionamiento de dichos servicios, hace aconsejable la creación de los Equipos Psicopedagógicos de Apoyo, que permitan una mayor operatividad cara a los centros educativos, al eliminar la concurrencia de alguna de sus funciones y al responder a planes de acción unitarios (Orden de 8 de agosto de 1.985, D.O.G. de 3 de septiembre)

\section{Normativa}

\begin{tabular}{|c|c|}
\hline \multicolumn{2}{|c|}{ SOBRE } \\
\hline Organización, funciones y regulación & Convocatoria de plazas \\
\hline $\begin{array}{l}\text { - Orden de 8/8/85 (D.O.G. de 3/9/85) } \\
\text { - Resolución de } 12 / 9 / 88 \text { (D.O.G. de } 15 / 9 / 88 \text { ) } \\
\text { - Circular no } 16 / 88 \text { de } 30 \text { de noviembre } \\
\text { - Circular } 8 / 92 \text { de } 1 \text { de junio } \\
\text { - Resolución de 26/11/96 (D.O.G. de 27/11/96) }\end{array}$ & $\begin{array}{l}\text { - Resolución de 29/4/86 (D.O.G. de 5/6/88) } \\
\text { - Resolución de 21/8/86 (D.O.G. de 2/9/86) } \\
\text { - Resolución de 26/8//86 (D.O.G. de 23/9/86) } \\
\text { - Resolución de } 30 / 10 / 87 \text { (D.O.G. de } 10 / 12 / 87 \\
\text { - Orden de } 7 / 4 / 89 \text { (D.OG. de } 22 / 5 / 89 \text { ) } \\
\text { - Orden de 5/9/89 (D.O.G. de } 2 / 10 / 89 \text { ) } \\
\text { - Orden de } 10 / 6 / 91 \text { (D.O.G. de } 3 / 7 / 91 \text { ) } \\
\text { - Orden de } 10 / 11 / 93 \text { (D.O.G. de } 13 / 12 / 93 \text { ) }\end{array}$ \\
\hline
\end{tabular}

\section{Niveles de actuación}

Los E.Ps.A. no atendían a Secundaria; su nivel de actuación ha sido preescolar y E.G.B. Posteriormente, con la entrada de la L.O.G.S.E., su actuación se centra en E. Infantil, E. Primaria y $7^{\circ}$ y $8^{\circ}$ de E.G.B.

Por la Resolución de 26 de noviembre de 1.996, el ámbito de intervención de los E.Ps.A. fueron los centros públicos de Educación Infantil, Primaria y Secundaria, incluyen- 
do a todos los alumnos de la enseñanza no universitaria y con carácter prioritario los de edades comprendidas entre los seis y los dieciseis años.

\section{Composición}

Los profesionales que componían los E.Ps.A. eran: Un Psicólogo, un Pedagogo y un Especialista en Audición y Lenguaje. En las siete grandes ciudades gallegas el número de miembros de los Equipos oscila entre siete y once; en alguna de estas ciudades se contaba con Asistente Social que, por regla general, procedían de los Equipos Multiprofesionales.

La provisión de puestos de Especialista en Audición y Lenguaje, se produce por primera vez en 1986, siendo sus funciones:

- Orientar al profesorado en el tratamiento de problemas de audición y lenguaje.

- Colaborar en la elaboración de programas de apoyo, dirigidos a la reeducación de dichas dificultades.

- Intervención directa en la rehabilitación de problemas de audición y lenguaje de los alumnos.

Estas funciones sufren pocas modificaciones con la Resolución de 26 de noviembre de 1996; así, en la segunda función se refiere a la colaboración en la elaboración de adaptaciones curriculares, en lugar de programas de apoyo.

En cada Equipo había un coordinador. Las funciones del coordinador, que fijaba la Resolución de 26 de noviembre, son las mismas que se contemplaban en la Circular 8/92, por la que se establece la figura del coordinador en los Equipos Psicopedagógicos de Apoyo.

La dirección de los E.Ps.A. a nivel provincial correspondía a un Inspector designado para ello. El control también le corresponde a la Inspección.

\section{Funciones}

Fijadas en la Orden de 8 de agosto de 1985.

- La prevención educativa en el medio escolar, familiar y social.

- La detección precoz de las deficiencias y trastornos en el desarrollo psicopedagógico del niño.

- La valoración pluridimensional de las necesidades y capacidades del alumno sujeto de Educación Especial.

- La elaboración de programas de desarrollo individual en base a las necesidades y capacidades de cada alumno y orientados a la solución de su problemática específica.

- El seguimiento del programa de desarrollo individual, en colaboración con el profesorado de los centros educativos.

- Dirigir o realizar, en su caso, las tareas de orientación escolar y vocacional de los alumnos, especialmente en los momentos críticos de su escolaridad y de su madurez psicobiológica.

- Asesorar y prestar ayuda técnica al profesorado en su función tutora. 
- Informar a padres, profesores y alumnos de las posibilidades de estudio y perspectivas profesionales.

- Colaborar en la elaboración del diseño de necesidades concretas del sector, que se elevará a través de la Inspección, a la Dirección General de Educación Básica.

- Coordinar las actividades de orientación que realicen los distintos departamentos de orientación de los colegios públicos, así como las que lleven a cabo, con autorización del Delegado Provincial de Educación respectivo, otros organismos en dichos centros.

\section{Convocatoria y selección}

Requisitos para el acceso

Pertenecer al cuerpo de profesores de Educación General Básica, con un mínimo de tres años de servicios, estando en posesión de alguna de las titulaciones siguientes:

- Licenciado en Filosofía y Letras (sección de Pedagogía) o en Filosofía y Ciencias de la Educación (sección de Pedagogía).

- Licenciado en Filosofía y Letras (sección de Psicología) o en Filosofía y Ciencias de la Educación (sección de Psicología).

- Licenciado en cualquier Facultad o Escuela Técnica Superior y Diplomado en Psicología Escolar.

En las dos primeras convocatorias no se exigía experiencia docente. Curiosamente, en estas primeras convocatorias existía el compromiso de residir en la localidad donde estaba situada la sede del servicio.

\section{Regulación de la Orientación Educativa y Profesional}

Se regula la orientación en Galicia, creando los Departamentos de Orientación en centros (IES y CEIP) y los Equipos de Orientación Específicos, como servicios externos, en cuyo plan de actuación estarán las Escuelas de EI, los IES, los CEIP, los CEP y los CEE.

\section{Normativa}

\begin{tabular}{|c|}
\hline SOBRE \\
\hline Organización, funciones y regulación \\
\hline - Decreto $120 / 1998$ de $23 / 4$ (D.O.G. de 27/4) \\
- Orden de 24/7/98 (D.O.G. de 31/7) \\
\hline
\end{tabular}

Ámbito de actuación

Los departamentos de orientación de los Institutos de Educación Secundaria y de los Centros Públicos Integrados, abarcarán en su plan de actuación, además del propio centro, 
aquellas Escuelas de Educación Infantil y Primaria y Colegios de Educación Primaria, que les estén adscritos.

Los departamentos de orientación de los Colegios de Educación Infantil y Primaria y de Educación Primaria, abarcarán en su plan de actuación, además del propio centro, aquellos otros centros incompletos de la misma zona de escolarización, que, en su caso, les adscriba la Delegación Provincial a propuesta del Servicio de Inspección Educativa.

Los equipos de orientación específicos abarcarán en su plan de actuación, las escuelas de educación infantil, los colegios de educación infantil y primaria, los colegios de educación primaria, los institutos de educación secundaria y los centros públicos integrados. Igualmente, los equipos de orientación específicos intervendrán en los centros específicos de educación de adultos y, en su caso, en los centros de educación especial, dependientes de la Administración Educativa.

\section{Composición}

Departamento de orientación de los Institutos de Educación Secundaria y de los Centros integrados

Formarán parte del departamento:

- Los funcionarios de carrera del cuerpo de profesores de enseñanza secundaria, de la especialidad de psicología y pedagogía, existentes en el centro.

- Los profesores o profesoras de pedagogía terapéutica y, en aquellos centros en los que esté catalogado, el especialista de audición y lenguaje que ejercen la función de apoyo a la atención del alumnado con necesidades educativas especiales, destinados en el instituto de educación secundaria.

- El jefe del departamento de orientación, si así fuera, de cada uno de los colegios de educación infantil y primaria y de educación primaria, adscritos al instituto de educación secundaria.

- Un tutor o tutora por cada uno de los ámbitos lingüístico-social y científico-tecnológico, designados por el director o directora, por propuesta de la comisión de coordinación pedagógica. En la designación se procurará que existan profesores de cada una de las etapas educativas que imparte el centro, o, cuando corresponda, de los distintos niveles educativos.

- En los institutos en los que se imparte formación profesional específica, se incorporará al departamento de orientación, un profesor o profesora que imparta el área de formación y orientación laboral, designado por el director/a, por propuesta de la comisión de coordinación pedagógica.

Departamento de orientación de los colegios de educación infantil y primaria y de educación primaria

- El jefe del departamento de orientación, que será un funcionario del cuerpo de maestros, preferentemente en posesión de la titulación de doctor o licenciado en psicopedagogía, o en psicología o en filosofía y ciencias de la educación (especialidades de psi- 
cología o ciencias de la educación) o en filosofía y letras (especialidades de pedagogía o psicología).

- El profesor/a de pedagogía terapéutica y de audición y lenguaje, que ejercen la función de apoyo a la atención del alumno con necesidades educativas especiales.

- Los coordinadores de ciclo y, si así fuera, un maestro/a de educación infantil, designado por la dirección, por propuesta de la comisión de coordinación pedagógica.

- Los maestros responsables, o la dirección de los colegios incompletos de la zona de escolarización que se determinen.

\section{Equipo de Orientación Específico}

Tendrá la composición que la Consellería de Educación y Ordenación Universitaria determine, en función de la evaluación de las necesidades educativas en las que la contextualizan las acciones docentes y, en todo caso, formarán parte de él:

- Un funcionario de carrera del cuerpo de profesores de enseñanza secundaria, de la especialidad de psicología y pedagogía, con la titulación de psicología o psicopedagogía.

- Un funcionario de carrera del cuerpo de profesores de enseñanza secundaria, de la especialidad de psicología o pedagogía, con la titulación de pedagogía o psicopedagogía.

- Un funcionario de carrera del cuerpo de maestros, especialista en audición y lenguaje.

- Un trabajador social y/o educador social.

La Consellería de Educación y Ordenación Universitaria posibilitará que, a nivel provincial, existan en estos equipos profesionales, cuando menos, con formación y experiencia en la orientación vocacional y profesional, en la atención de alumnado con discapacidades sensoriales, motrices, sobredotación intelectual, trastornos generalizados del desenvolvimiento y trastornos de conducta.

\section{Funciones}

- Colaborar, como técnicos educativos, en las diferentes actuaciones encaminadas a la prevención y atención temprana que faciliten al alumnado su desarrollo evolutivo.

- Asesorar y apoyar a los departamentos de orientación, de los centros de la provincia, mediante la elaboración de pautas de intervención, la intervención directa, el seguimiento de las acciones iniciadas y cualquier otra acción o prestación de recursos que se precise.

- Desarrollar programas de investigación, elaboración, recopilación y difusión de recursos, para dar respuesta a las necesidades específicas del alumnado.

- Asesorar a los departamentos de orientación de los centros educativos en la atención a la diversidad personal y sociocultural del alumnado y colaborar en la aplicación de medidas educativas apropiadas.

- Colaborar con los departamentos de orientación en la evaluación psicopedagógica del alumnado, cuando sea necesaria la intervención de un profesional externo al centro y participar, en su caso, en el dictamen de escolarización. 
- Contribuir a la formación especializada de los departamentos de orientación y del profesorado en el ámbito de las necesidades educativas especiales que atienden.

- Colaborar con otros servicios e instituciones que desarrollen actividades en el campo educativo, laboral, social, de la salud y en aquellos otros ámbitos de su competencia.

- Cooperar con los departamentos de orientación de los centros de su ámbito de actuación en el diseño, desarrollo y evaluación de programas de intervención relativos a la orientación profesional, a la modificación de la conducta, a la salud, etc. o de programas integrados.

- Colaborar en el diseño, desarrollo y evaluación de actividades o en programas formativos de orientación educativa y sociofamiliar, así como asesorar y colaborar con las familias sobre el tipo de intervención a seguir con sus hijos.

- Cualquier otra que se les pudiera encomendar, en el ámbito de sus actuaciones.

\section{Organización del trabajo}

Departamentos de Orientación

a) Funcionamiento

Los miembros del departamento de orientación con destino en el propio centro, celebrarán reuniones semanales en la hora destinada para tal fin en su horario. La asistencia será obligatoria.

b) El maestro responsable de la orientación en los centros en los que no exista departamento de orientación, recogerá en un informe anual la evaluación del desarrollo del plan de orientación y las modificaciones adoptadas, en su caso, que se incorporará al anexo de la memoria final del instituto de educación secundaria o centro público integrado, al que esté adscrito.

c) Elaboración y estructura del plan de orientación y de su memoria

El plan de orientación incluirá, al menos, los siguientes aspectos:

- Justificación basada en el contexto.

- Objetivos.

- Planificación general y definición de acciones prioritarias.

- Estrategias de intervención.

- Criterios de evaluación del plan.

Equipo de Orientación Específico

a) Organización y funcionamiento.

- El Equipo de Orientación Específico estará coordinado por uno de sus miembros, que será el responsable de la unificación de criterios técnicos de intervención, entre ellos.

- Deberá establecer diferentes canales de comunicación que permitan la colaboración, en el ámbito de sus competencias, con los diferentes agentes de la comuni- 
dad educativa, así como con otros servicios o instituciones que posibiliten actuaciones conjuntas y coherentes con los objetivos previstos en cada contexto educativo.

- Celebrarán, dentro de su horario, una reunión semanal de coordinación, siendo por lo tanto de asistencia obligatoria para todos sus miembros. Al menos una vez al mes, las reuniones de los Equipos tendrán por objeto hacer el seguimiento de desarrollo del plan de actuación y establecer las medidas de reajuste que, en su caso, se estimen necesarias.

- A nivel provincial, le corresponde la coordinación del Equipo de Orientación Específico a un Inspector de Educación.

El plan anual será aprobado por el Delegado provincial y el seguimiento será realizado por el Inspector coordinador correspondiente.

Los miembros de los Equipos de Orientación Específicos, desempeñarán sus funciones a través de un horario de trabajo que permita el contacto con la comunidad educativa de los centros.

Cada miembro del Equipo de Orientación específico, dedicará un día a la semana, en la sede correspondiente, y en horario de mañana y tarde, a la atención personalizada de las diferentes demandas socioeducativas de su ámbito de actuación.

Todos los miembros del Equipo de Orientación Específico, deberán permanecer en la sede, al menos, una jornada de mañana o de tarde para facilitar la atención coordinada a la comunidad educativa.

b) Plan de actuación y memoria.

Corresponde a cada Equipo la elaboración del plan de actuación anual y la realización del seguimiento de éste. El mencionado plan deberá someterse, antes del 15 de Octubre, a la aprobación del Inspector coordinador correspondiente y será remitido a la Subdirección General de Ordenación Educativa, con el visto bueno del Delegado provincial.

\section{Conclusión}

Hasta aquí hemos visto que la orientación cobra, en nuestro país, una relevancia especial, desde que en 1987 y 1989, aparecen publicados el Proyecto de Reforma de la Enseñanza y el Libro Blanco para la Reforma Educativa, que finalizan con la promulgación de la LOGSE en 1.990. Desde esa fecha hasta la actualidad, se han realizado numerosos análisis sobre el planteamiento de la orientación en la Reforma.

También se generaron debates sobre los principios básicos o modelos de intervención, organización y funcionamiento de los profesionales encargados, culminando con el documento publicado por el M.E.C. (1.990) titulado La Orientación Educativa y la intervención psicopedagógica.

Vemos que la orientación educativa, constituye un derecho del alumno, ya que la docencia no se agota con la transmisión de conceptos y el objetivo consiste en contribuir al desenvolvimiento personal. Este derecho, se garantiza a través de un conjunto de servicios y 
actividades que ofrece el sistema educativo en las distintas Comunidades y, entre ellas, en la Comunidad Autónoma de Galicia como hemos visto.

\section{Legislación}

CONSELLERÍA DE EDUCACIÓN E ORDENACIÓN UNIVERSITARIA: Resolución de 15 de diciembre de 1982 (Diario Oficial de Galicia, de 14 de febrero) por la que se regula el funcionamiento de los SOEV.

CONSELLERÍA DE EDUCACIÓN E ORDENACIÓN UNIVERSITARIA: Resolución de 11 de mayo de 1983 (Diario Oficial de Galicia de 17 de junio) por la que se regula la composición, organización y funciones de los Equipos Multiprofesionales

CONSELLERÍA DE EDUCACIÓN E ORDENACIÓN UNIVERSITARIA: Resolución de 26 de Noviembre de 1996 (Diario Oficial de Galicia de 27 de Noviembre) por la que se determinan las funciones y se regula el plan de actividades y el régimen de funcionamiento de los EPsA de la Comunidad Autónoma de Galicia.

CONSELLERÍA DE EDUCACIÓN E ORDENACIÓN UNIVERSITARIA: Decreto 120/1998, de 23 de abril (Diario Oficial de Galicia de 27 de abril) por el que se regula la orientación educativa y profesional en la Comunidad Autónoma de Galicia.

CONSELLERÍA DE EDUCACIÓN E ORDENACIÓN UNIVERSITARIA: Orden de 24 de julio de 1998 (Diario Oficial de Galicia de 31 de julio) por la que se establece la organización y funcionamiento de la orientación educativa y profesional en la Comunidad Autónoma de Galicia.

MINISTERIO DE EDUCACIÓN Y CIENCIA. (1987): Proyecto para la Reforma de la Enseñanza. Madrid.

MINISTERIO DE EDUCACIÓN Y CIENCIA. (1989): Libro Blanco para la Reforma del Sistema Educativo. Madrid.

MINISTERIO DE EDUCACIÓN Y CIENCIA. Ley Orgánica 1/1990, de 3 de octubre, de Ordenación General del Sistema Educativo. (Boletín Oficial del Estado de 4 de octubre). Madrid

MINISTERIO DE EDUCACIÓN Y CIENCIA. (1990): La orientación educativa y la intervención psicopedagógica. Madrid.

MINISTERIO DE EDUCACIÓN Y CIENCIA. (1991): El sistema Educativo español. CIDE. Madrid.

MINISTERIO DE EDUCACIÓN Y CIENCIA. (1992): Orientación y tutoría. Madrid.

MINISTERIO DE EDUCACIÓN Y CIENCIA. (1989): Real Decreto 850/1993 del 14 de julio (Boletín Oficial del Estado de 20 de julio), por el que se regula la provisión de puestos de trabajo en centros públicos. Madrid.

Fecha de recepción: 14-01-2003

Fecha de revisión: 18-09-2004

Fecha de aceptación: 05-11-2004 